\section{Screening mutant libraries of T7 RNA polymerase for candidates with increased acceptance of $\mathbf{2}^{\prime}$-modified nucleotides $\dagger$}

\author{
Vanessa Siegmund, ${ }^{a}$ Tobias Santner, ${ }^{b}$ Ronald Micura ${ }^{b}$ and Andreas Marx ${ }^{* a}$ \\ Received 13th July 2012, Accepted 13th August 2012 \\ DOI: $10.1039 / \mathrm{c} 2 \mathrm{cc} 35028 \mathrm{a}$
}

We present a screening assay based on fluorescence readout for the directed evolution of T7 RNA polymerase variants with acceptance of $2^{\prime}$-modified nucleotides. By using this screening we were able to identify a T7 RNA polymerase mutant with increased acceptance of $2^{\prime}$-methylseleno- $2^{\prime}$-deoxyuridine $5^{\prime}$-triphosphate.

Modified RNA plays a key role in many biochemical and biophysical techniques for the investigation of RNA structures and function and for use in cutting edge technologies as an aptamer or siRNA. ${ }^{1}$ Among several positions for site-specific labeling of RNA, the $2^{\prime}$-position of the sugar moiety is of major interest, since modifications can be incorporated in both purine and pyrimidine nucleosides. $2^{\prime}$-Modified RNA is used for technologies in which increased nuclease resistance and chemical stability are of great importance and in which naturally occurring nucleic acids have limitations. ${ }^{2}$ Furthermore, the $2^{\prime}$-ribose position has been shown to be advantageous for the introduction of selenium into RNA for use in X-ray crystallography. ${ }^{3}$ In addition to commonly used chemical solid phase synthesis of modified RNA using phosphoramidite building blocks, the enzymatic synthesis of modified RNA using T7 RNA polymerase and modified ribonucleoside 5 -triphosphates (NTPs) has been more and more investigated over the past few years. The demand for T7 RNA polymerase mutants generated by protein engineering that are able to incorporate modified nucleotides more efficiently therefore raises the need for new screening systems for selection of interesting candidates from mutant libraries.

Directed protein evolution as an iterative process of mutagenesis, expression and screening or selection has been successfully used to establish DNA polymerases with altered properties like the ability to incorporate ribonucleotides, ${ }^{4}$ modified nucleotides like $2^{\prime}$ - $O$-methyl ribonucleotides ${ }^{5}$ or with increased reverse transcriptase activity $^{6}$ for a variety of applications. Several reported selection methods like phage display $^{7}$ or

\footnotetext{
${ }^{a}$ Department of Chemistry and Konstanz Research School Chemical Biology, University of Konstanz, Universitätsstraße 10, 78457 Konstanz, Germany.E-mail: andreas.marx@uni-konstanz.de; Fax: + 49 7531-885140; Tel: + 49 7531-885139

${ }^{b}$ Institute of Organic Chemistry, Center for Chemistry and Biomedicine, Leopold-Franzens University, Innrain 80-82, 6020 Innsbruck, Austria

$\dagger$ Electronic supplementary information (ESI) available. See DOI: $10.1039 / \mathrm{c} 2 \mathrm{cc} 35028 \mathrm{a}$
}

compartmentalized self-replication ${ }^{8}$ have been developed and different screening platforms based on fluorescence readout of synthesized DNA have been established to evolve DNA polymerase mutants with altered properties. ${ }^{9}$ Regarding the expansion of the substrate spectra of RNA polymerases, only a few approaches based on rational design and site-directed mutagenesis of T7 RNA polymerase have been reported so far. ${ }^{10}$ Recently, the directed evolution of a T7 RNA polymerase mutant, referred to as 'RGVG' E593G, V685A, with increased acceptance for $2^{\prime}-O$-methyl NTPs (2'-OMe-NTPs) was reported. ${ }^{11}$

Previously, we have investigated the applicability of two T7 RNA polymerase mutants for the enzymatic synthesis of $2^{\prime}$-methylseleno ( $2^{\prime}$-SeMe) modified RNA. ${ }^{12}$ We demonstrated the efficient enzymatic synthesis of $2^{\prime}$-SeMe-modified RNA using mutant 'RGVG' E593G, V685A and thus provided a foundation for an alternative derivatization strategy for RNA structure determination using the multiwavelength anomalous dispersion technique (MAD). Encouraged by our previous findings, we here report the screening of T7 RNA polymerase mutants with increased acceptance of $2^{\prime}$-SeMe-UTP and $2^{\prime}$-OMe-UTP.

We started with the construction of a T7 RNA polymerase mutant library by randomizing amino acid positions Y639 and $\mathrm{H784}$. Both residues are located in the active site of the polymerase and have been shown to be involved in ribose recognition events and nucleotide discrimination. ${ }^{13}$ DNA fragments containing each of the randomized regions were prepared by PCR using oligonucleotide primers in which the codons of amino acids Y639 and H784 were randomized. The fragments were purified and assembled in a second step by overlap extension PCR. Afterwards, the assembled PCR product was cloned into a vector (pGDR11) suitable for protein expression in $E$. coli BL21 cells under control of an IPTG (isopropyl- $\beta$-D-thiogalactopyranoside) inducible T5 promotor. Electrocompetent E. coli BL21 cells were transformed with the mutant library and codon randomization was verified by sequencing the T7 RNA polymerase gene of several transformants. We picked 3200 transformants and cultivated them in 384-deepwell plates for further storage at $-80{ }^{\circ} \mathrm{C}$. The mutants from the library were expressed, E. coli cells harvested and lysed using lysozyme in 96-well plates as previously described for the screening of DNA polymerases. ${ }^{9}$ Cell lysates containing the overexpressed T7 RNA polymerase mutants were diluted with buffer and directly used for activity screening. 
A)

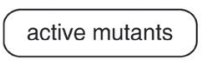

B)

inactive mutants

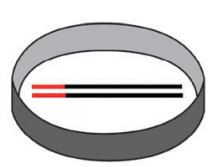

dsDNA template with T7 promotor sequence

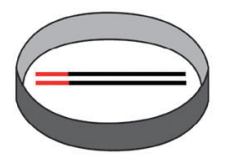

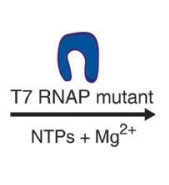

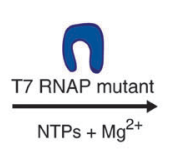

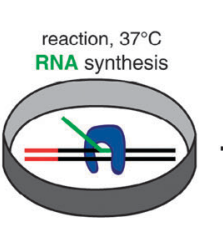

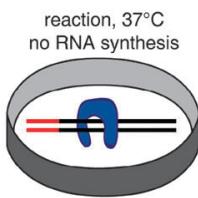

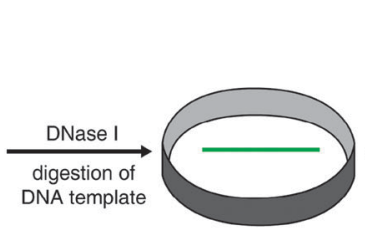

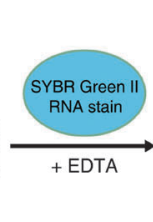

fluorescence signal
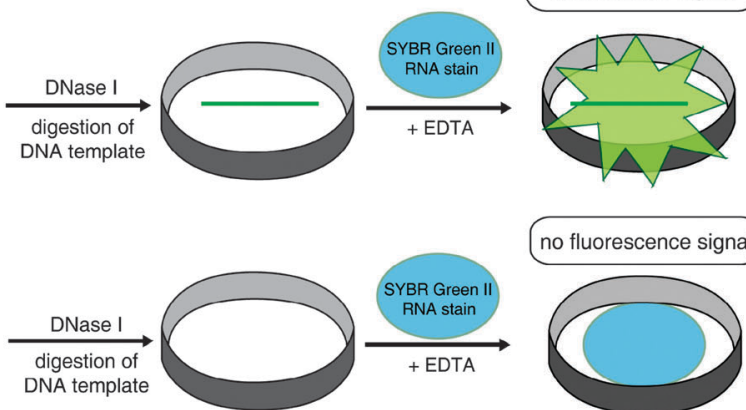

Fig. 1 General scheme of the screening approach for T7 RNA polymerase activity. Enzyme variants that have RNA polymerase activity (A) are identified with the RNA-specific dye SYBR Green II while inactive mutants (B) are not able to generate a fluorescence signal.

For screening of the T7 RNA polymerase mutant library with highthroughput, we established a screening assay for the detection of polymerase activity directly from diluted $E$. coli lysates in 384-well plates. This assay is based on in vitro transcription using a double stranded DNA template with the T7 promotor sequence coding for a 89 nt long transcript, NTPs and transcription buffer containing $\mathrm{MgCl}_{2}$ for enzyme activity. After incubating the reaction mixture with the lysates at $37^{\circ} \mathrm{C}$ to initiate RNA synthesis in the presence of active mutants, the DNA template was digested using DNase I and the assay afterwards stopped by the addition of a buffered solution containing EDTA and SYBR Green II, a RNA specific stain for subsequent fluorescence readout of synthesized RNA using a microplate reader (Fig. 1). A similar screening assay was reported by Kuhlman et al. ${ }^{14}$ and is also commercially available from Profoldin. However, omitting the ultrafiltration step required in the published assay and the cost-intensive RNase inhibitor simplifies our assay and renders it better suited for screening libraries with high-throughput. To distinguish between active and inactive mutants, E. coli cells containing the T7 RNA polymerase wild type plasmid were used as positive control and E. coli cells harboring the empty pGDR11 vector as negative control. These controls were expressed along with the mutants in 96-well plates and were present on all 384-well assay plates during the screening process. Fluorescence measurements revealed a low background activity and showed a considerable signal-to-noise ratio of about $1: 10$ for wild type T7 RNA polymerase and of about $1: 2$ for T7 RNA polymerase mutant 'RGVG' E593G, V685A that was used as control for screening the second mutant library. The significant lower signal-to-noise ratio for mutant 'RGVG' E593G, V685A derives from its lower activity compared to the wild type T7 RNA polymerase when natural NTPs are assayed. It is worth noting that an automated pipetting device and the fluorescence readout allowed the screening and evaluation of 384 reactions in approximately $3 \mathrm{~h}$ including the reaction time of $90 \mathrm{~min}$.

Out of the 3200 screened mutants, we identified 55 active variants that showed at least $30 \%$ activity in comparison to the positive control using the wild type enzyme as determined by fluorescence readout (Fig. S1, ESI $\dagger$ ). These 55 hits were again expressed in E. coli and the T7 RNA polymerase mutants purified via the $6 \times$ histidine tag by affinity chromatography in 96-well plates. Wild type T7 RNA polymerase and mutant 'RGVG' E593G, V685A were purified along with the mutants to ensure homogeneity during purification. The 55 mutants were adjusted to the same concentration and afterwards tested in ${ }^{32} \mathrm{P}$-based in vitro transcription assays for activities using NTPs. Additionally, the mutants were tested for an increased acceptance of $2^{\prime}$-OMe- and $2^{\prime}$-SeMeUTP in comparison to wild type T7 RNA polymerase and in comparison to the mutant 'RGVG' E593G, V685A. The transcription reactions were analyzed by denaturing polyacrylamide gel electrophoresis (PAGE) and phosphorimaging. Thereby, we validated the activity of all 55 tested mutants in reactions with all natural NTPs verifying that our screening system is suitable to screen T7 RNA polymerase libraries for active mutants (data not shown). We could also identify 10 mutants that were able to incorporate $2^{\prime}$-OMe-UTP with increased efficiency and $2^{\prime}$-SeMe-UTP with moderate but reasonable efficiencies by using ${ }^{32} \mathrm{P}$-based in vitro transcription assays as described above. The genes of these 10 selected mutants were sequenced and analyzed revealing that these hits were composed of only three different variants: the double mutant $\mathrm{Y} 639 \mathrm{~F} / \mathrm{H} 784 \mathrm{~A}$, the mutant $\mathrm{Y} 639 \mathrm{~F}$ and the mutant Y639M. Interestingly, these mutants have already been characterized and described for their increased acceptance of $2^{\prime}$-modified NTPs. ${ }^{10,11}$ PAGE gel analysis indicated that the 3 mutants can incorporate the $2^{\prime}$-OMe- and $2^{\prime}$-SeMemodification into an $89 \mathrm{nt}$ long transcript, but often stall at positions where uridine has to be incorporated resulting in a mixture of truncated transcripts (Fig. S2, ESI $\dagger$ ). Thereby, less full length product was formed in comparison to reactions with mutant 'RGVG' E593G, V685A that can synthesize modified full length transcripts without stalling.

Encouraged by these results, we constructed a second mutant library by randomizing the gene of mutant ' $R G V G$ ' E593G, V685A by error-prone PCR. Thereby, the gene of the enzyme was arbitrarily randomized in order to identify new amino acid positions that might effect processing of $2^{\prime}$-modified nucleotides. The randomized T7 RNA polymerase mutant genes were cloned into the pGDR11 vector and E. coli BL21 cells were transformed with the mutant library. A total number of 1600 transformants were picked, cultivated, expressed and screened as described above. By doing so, we could identify 38 active hits that showed at least the same level of activity as the parental mutant (Fig. S3, ESI $\dagger$ ). The hits were afterwards expressed, purified and tested in ${ }^{32} \mathrm{P}$-based in vitro transcription assays for activities. We identified a mutant that showed incorporation efficiencies comparable to the parental mutant 'RGVG' E593G, V685A when NTPs and 2'-OMe-UTP were 


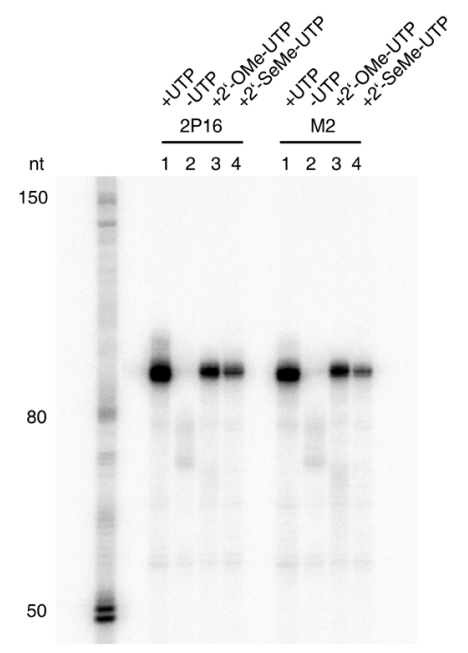

Fig. 2 Transcription assays with $2^{\prime}$-modified UTP using mutant $2 \mathrm{P} 16$. Activity of the mutant was compared to reactions with T7 RNA polymerase mutant 'RGVG' E593G, V685A (M2). Transcripts were labeled by the inclusion of $\left[\alpha^{32} \mathrm{P}\right] \mathrm{GTP}$ and analyzed by denaturing PAGE. Lane 1: transcription in the presence of ATP, CTP, GTP and UTP; lane 2: the same as lane 1, but no UTP; lane 3: the same as lane 2, but with 2'-OMe-UTP; lane 4: the same as lane 2, but with $2^{\prime}$-SeMe-UTP.

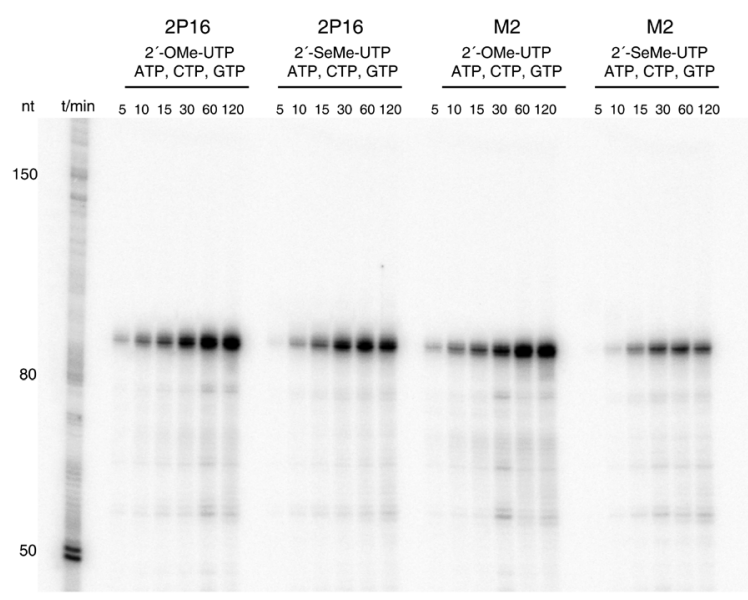

Fig. 3 Time-course experiment for the incorporation of 2'-OMeUTP and $2^{\prime}$-SeMe-UTP into RNA by T7 RNA polymerase mutants 2P16 and mutant 'RGVG' E593G, V685A (M2). The 89 nt long transcripts were labeled by the inclusion of $\left[\alpha^{32} \mathrm{P}\right] \mathrm{GTP}$ and analyzed by denaturing PAGE. Aliquots of the reaction mixtures were withdrawn at the corresponding time points and quenched with an equal volume of stop solution containing $50 \mathrm{mM}$ EDTA.

assayed as substrates (Fig. 2, lanes 1 and 3), as demonstrated by PAGE gel analysis. This mutant was referred to as $2 \mathrm{P} 16$. When only ATP, CTP and GTP were added to the reaction mixture, no product formation was observed (Fig. 2, lane 2). In reactions where $2^{\prime}$-SeMe-UTP was present together with ATP, CTP and GTP (Fig. 2, lane 4), we observed increased incorporation efficiencies of the 2 '-SeMe-modification into the 89 nt long transcript. These results were confirmed by a timecourse experiment in which samples were withdrawn from the reaction mixture at different time points between 5 min and $2 \mathrm{~h}$ and immediately stopped (Fig. 3). Comparing total intensities of the synthesized full-length products after $2 \mathrm{~h}$ of transcription revealed that mutant $2 \mathrm{P} 16$ was able to synthesize approximately twice as much of the $2^{\prime}$-SeMe-modified RNA as the parental mutant.

Sequencing of mutant $2 \mathrm{P} 16$ revealed that this mutant possesses 7 additional mutations (I119V, G225S, K333N, D366N, F400L, S661G, F880Y) distributed all over the RNA polymerase gene along with the initial mutations. Surprisingly, this number of additional mutations did not change the polymerase's ability to use normal ribonucleotides and $2^{\prime}$-OMe-UTP as substrates, but enhances the ability to use $2^{\prime}$-SeMe-UTP.

In summary, we have established a high-throughput assay for screening T7 RNA polymerase mutant libraries based on fluorescence readout of synthesized RNA. The assay was successfully used to identify active T7 RNA polymerase mutants from two different libraries. After expression and purification of these mutants, we could identify a candidate, referred to as $2 \mathrm{P} 16$, that showed comparable activities to the parental mutant 'RGVG' E593G, V685A when normal NTPs and $2^{\prime}$-OMe-UTP were assayed, and even slightly better incorporation efficiencies for $2^{\prime}$-SeMe-UTP. These results render our established screening platform promising for identifying RNA polymerases with increased acceptance for modified nucleotides and altered properties, as well as the discovery of RNA polymerase inhibitors.

We gratefully acknowledge support from the DFG (MA 2288/12) and FWF (I317).

\section{Notes and references}

1 (a) M. A. Behlke, Oligonucleotides, 2008, 18, 305-319; (b) J. K. Watts, G. F. Deleavey and M. J. Damha, Drug Discovery Today, 2008, 13, 842-855; (c) G. Mayer, Angew. Chem., Int. Ed., 2009, 48, 2672-2689.

2 (a) B. E. Eaton and W. A. Pieken, Annu. Rev. Biochem., 1995, 64 837-863; (b) S. P. Zinnen, K. Domenico, M. Wilson, B. A. Dickinson, A. Beaudry, V. Mokler, A. T. Daniher, A. Burgin and L. Beigelman, $R N A, 2002,8,214-228$.

3 (a) C. Höbartner and R. Micura, J. Am. Chem. Soc., 2004, 126, 1141-1149; (b) V. Olieric, U. Rieder, K. Lang, A. Serganov, C. Schulze-Briese, R. Micura, P. Dumas and E. Ennifar, $R N A$, 2009, 15, 707-715; (c) A. Serganov, S. Keiper, L. Malinina, V. Tereshko, E. Skripkin, C. Höbartner, A. Polonskaia, A. Tuân Phan, R. Wombacher, R. Micura, Z. Dauter, A. Jäschke and D. J. Patel, Nat. Struct. Mol. Biol., 2005, 12, 218-224; (d) L. Lin, J. Sheng and Z. Huang, Chem. Soc. Rev., 2011, 40, 4591-4602.

4 (a) N. Staiger and A. Marx, ChemBioChem, 2010, 11, 1963-1966; (b) C. Cozens, V. B. Pinheiro, A. Vaisman, R. Woodgate and P. Holliger, Proc. Natl. Acad. Sci. U. S. A., 2012, 109, 8067-8072.

$5 \mathrm{M} . \mathrm{Fa}$, A. Radeghieri, A. A. Henry and F. E. Romesberg, J. Am. Chem. Soc., 2004, 126, 1748-1754.

6 K. B. Sauter and A. Marx, Angew. Chem., Int. Ed., 2006, 45, 7633-7635.

7 S. Vichier-Guerre, S. Ferris, N. Auberger, K. Mahiddine and J. L. Jestin, Angew. Chem., Int. Ed., 2006, 45, 6133-6137.

8 M. d'Abbadie, M. Hofreiter, A. Vaisman, D. Loakes, D. Gasparutto, J. Cadet, R. Woodgate, S. Paabo and P. Holliger, Nat. Biotechnol., 2007, 25, 939-943.

9 (a) D. Summerer, N. Z. Rudinger, I. Detmer and A. Marx, Angew. Chem., Int. Ed., 2005, 44, 4712-4715; (b) C. Gloeckner, K. B. Sauter and A. Marx, Angew. Chem., Int. Ed., 2007, 46, 3115-3117.

10 (a) R. Padilla and R. Sousa, Nucleic Acids Res., 1999, 27, 1561-1563; (b) R. Padilla and R. Sousa, Nucleic Acids Res., 2002, 30, e138.

11 J. Chelliserrykattil and A. D. Ellington, Nat. Biotechnol., 2004, 22, $1155-1160$.

12 V. Siegmund, T. Santner, R. Micura and A. Marx, Chem. Sci., 2011, 2, 2224-2231.

13 (a) L. G. Brieba and R. Sousa, Biochemistry, 2000, 39, 919-923; (b) Y. Huang, F. Eckstein, R. Padilla and R. Sousa, Biochemistry, 1997, 36, 8231-8242.

14 P. Kuhlman, H. L. Duff and A. Galant, Anal. Biochem., 2004, 324, 183-190. 